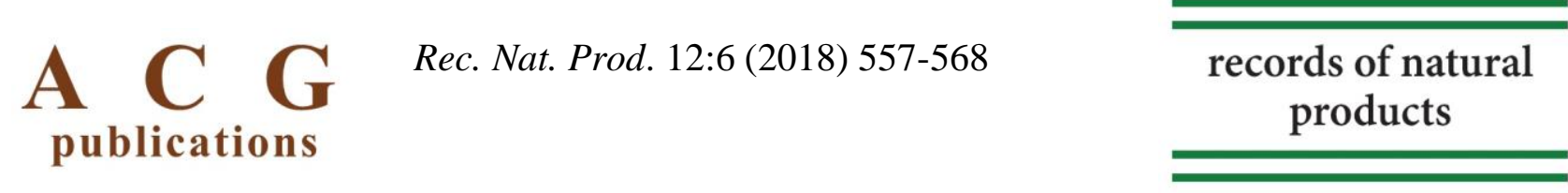

\title{
Investigation of Pesticidal Activities of Essential Oil of Eucalyptus camaldulensis Dehnh
}

\author{
Tamer Üstüner $^{\oplus 1, *}$, Şaban Kordali ${ }^{\oplus 2}$, Ayșe Usanmaz Bozhüyük ${ }^{\oplus 3}$ and \\ Memiş Kesdek $^{\oplus 4}$
}

\author{
${ }^{1}$ Plant Protection Department, Agriculture Faculty, University of Kahramanmaras Sutcu Imam, \\ Kahramanmaras, Türkiye \\ ${ }^{2}$ Plant Protection Department, Agriculture Faculty, University of Atatürk, Erzurum, Türkiye \\ ${ }^{3}$ Plant Protection Department, Agriculture Faculty, University of Igdir, Igdir, Türkiye \\ ${ }^{4}$ Fethiye A.S.M. Kocman High school, University of Mugla Sitkı Kocman, Mugla, Türkiye
}

(Received January 22, 2018; Revised March 20, 2018; Accepted March 21, 2018)

\begin{abstract}
In this study, chemical compositions of the volatile oil extracted from Eucalyptus camaldulensis Dehnh. were analyzed by using GC and GC-MS. The oxygenated sesquiterpenes, monoterpene hydrocarbons, sesquiterpene hydrocarbons and oxygenated monoterpenes compositions were detected in the sample studied. Pesticidal effects of this oil were investigated on storage insect pests like Rhizopertha dominica F. (Col.: Bostrychidae), Sitophilus granarius L. (Col.: Curculionidae), Tribolium confusum Duv. (Col.: Tenebrionidae), Callosobruchus maculatus F. and Acanthoscelides obtectus Say. (Col.: Bruchidae). The essential oil was tested on some fungal pathogens and weeds. As fungal pathogens, Verticillium dahliae Kleb, Fusarium oxyporum Schl., Phytium debaryanum Auct. non R. Hesse, Sclerotinia sclerotiorum (Lib.) de Barry and Rhizoctania solani Kühn. were used while tests on the weeds were performed on Convolvulus arvensis L., Melilotus officinalis L. and Amaranthus retroflexus L. in invitro conditions. E. camaldulensis essential oil was found to be effective at 10 and $20 \mu \mathrm{L}$ against all the tested insect pests. Our results also showed that growth of fungal mycelial as well as weed stems and roots were significantly affected by essential oil. In 10 and $20 \mu \mathrm{L}, V$. dahliae, P. debaryanum, F. oxyporum and S. sclerotiorum mycelial growth were inhibited in 7 days, while no effect was observed on $R$. solani mycelial growth in this duration. On the other hand, the applications of the oil to the weeds showed different results for each species examined. Although at 5,10 and $20 \mu \mathrm{L}$ concentrations of E. camaldulensis essential oil did not affect the root and stem growth of $C$. arvensis, the stem and root growth of $M$. officinalis and A. retroflexus were reduced by the tested essential oil at the same concentration and time. The research results suggest that E. camaldulensis essential oil might have potential to be used as a natural pesticide as well as fungicide.
\end{abstract}

Keywords: Eucalyptus camaldulensis Dehnh.; essential oil; pesticidal effect. @ 2018 ACG Publications. All rights reserved.

\section{Introduction}

In recent years, scientists have focused on the ways to increase the food production because of the fast growing demand related to the growth of the world population. Unfortunately, substantial yield losses in food products occur due to insects and plant diseases [1]. Despite the fact that there are several methods for pest control including mechanical, chemical and biological approaches [2]. The materials used in these techniques may leave cause toxic residues in treated crops. Synthetic pesticides in particular can cause serious environmental pollution owing to their slow biodegradation. Besides, it has been shown that the

\footnotetext{
* Corresponding author: E-Mail: tamerustuner@ksu.edu.tr; Fax: 0344.3002002
} 
intensive use and long term use of pesticide has resulted in development of chemical resistance amongst insects, disease and weeds [3, 4]. Consequently, scientists around the globe have focused on finding new potential biological pesticides which will show different selective insecticidal mechanisms in comparison to synthetic chemicals [5-7]. Esential oils obtained from different plants (Pimpinella anisum, E. camaldulensis Dehnh., Eucalyptus globulus and Satureja thymbra) have been demonstrated to have different mortality rates on different pest such as Tribolim confusum Duv., T. castaneum, Sitophilus oryzae, S. granarius L., C. maculatus F. and A. obtectus [8-10].

It was recorded that the essential oil obtained from plants such as E. camaldulensis, E. unigera and E. globulus had toxic effects on fungi like Colletotrichum gloeosporioides, Fusarium moniliforme, $F$. oxysporum, F. solani, Pythium spp., P. ultimum and R. solani [11-14].

Another important problem is weeds in agricultural areas. Albeit an effective method to eliminate the undesirable herbs in the field, intensive use of synthetic herbicides can result in soil and groundwater contamination, and development of weed resistance. A great number of research has been conducted about E. camaldulensis essential oils to prevent germination of many weeds as A. retroflexus, Chenopodium album, Cyperus rotundus and Solanum nigrum in the cultivated sites [4, 15-20]. Adverse effects of essential oil of E. camaldulensis were determined on the germination and seedling growth of many species of weed including Amaranthus hybrid, A. retroflexus, C. album, Cirsium arvense, Rumex crispus and Portulaca oleracea [3, 21].

In the present study, we aimed at evaluating the pesticidal effect of the essential oil isolated from $E$. camaldulensis Dehnh. on some stored product pests, fungi and weeds.

\section{Material and Methods}

\subsection{Plant Materials and Isolation of Essential Oils}

E. camaldulensis leaves were collected from Tarsus region of Turkey between June and August of 2016. Tarsus located in the Latitude $36^{\circ} 54^{\prime} 59.62^{\prime \prime} \mathrm{N}$ and longitude $34^{\circ} 53^{\prime} 42.76^{\prime \prime} \mathrm{E}$, and its annual average temperature is $26.7^{\circ} \mathrm{C}$, while its altitude reaches about $23 \mathrm{~m}$, the region also has clay-sandy soil. The samples collected from the region were sent to the herbarium laboratory, Department of Plant Protection, Faculty of Agriculture, Atatürk University, Erzurum, Turkey, where they were dried in shade and ground in a grinder. The dried samples $(500 \mathrm{~g})$ were subjected to hydrodistillation for $4 \mathrm{~h}$ using a Clevenger-type apparatus. The hydrodistillation of E. camaldulensis $1.5 \%(\mathrm{w} / \mathrm{w})$ yielded the accumulations of its essential oil. Once obtained, the essential oil was stored at $4{ }^{\circ} \mathrm{C}$ in a fridge for further tests.

\subsection{GC and GC-MS Analysis}

The analysis of the essential oil was performed with a Thermofinnigan Trace GC-FID and GC/Trace DSQ/A1300 (E.I. Quadrapole) equipped with a SGE-BPX5 MS fused silica capillary column (30 $\mathrm{m} \times 0.25 \mathrm{~mm}$ i.d., film thickness $0.25 \mu \mathrm{m}$ ). For GC-MS detection, an electron impact ionization system with ionization energy of $70 \mathrm{eV}$ was used. Carrier gas was Helium at a flow rate of $1.0 \mu \mathrm{L} / \mathrm{min}$. diluted samples $(1 / 100, v / v$, in methylene chloride) of was injected in the splitless mode. Injector and MS transfer line temperatures were set at $220^{\circ} \mathrm{C}$ and $290^{\circ} \mathrm{C}$, respectively (Table 1). The oven temperature was programmed to raise from $50^{\circ} \mathrm{C}$ to $150^{\circ} \mathrm{C}$ at $3^{\circ} \mathrm{C} / \mathrm{min}$, then to hold isothermal for $10 \mathrm{~min}$. and finally raised to $250^{\circ} \mathrm{C}$ at $10^{\circ} \mathrm{C} / \mathrm{min}$.

\subsection{Insect Material}

$R$. dominica, S. granarius, T. confusum, C. maculatus and A. obtectus adults were collected from private store houses in Erzurum/Turkey and kept on cowpea (the black-eyed pea), wheat grains, cracked grains, flour and kidney beans seeds depending on the species studied. The cultures were maintained in Department of Plant Protection, Faculty of Agriculture, Atatürk University, Erzurum, Turkey. In addition, the cowpea, wheat and kidney bean seeds were purchased from a local market and kept at $-15{ }^{\circ} \mathrm{C}$ in a freezer in order to avoid any arthropod pests contamination prior to use for bioassay during two days. $C$. maculatus and A. obtectus adults were reared in 1-L jars containing cowpea and kidney bean seeds, whilst 
$R$. dominica, S. granarius and T. confusum adults were reared in 1-L jars containing wheat grains, cracked grains and uninfected flour respectively. The cultures were maintained in the dark conditions in a growth chamber set at $25 \pm 2{ }^{\circ} \mathrm{C}$ and $65 \pm 5 \% \mathrm{rh}$. without exposure to any insecticide for several generations. Adult insects (three day-old) were used for the fumigant toxicity test. All experimental procedures were carried out under the same environmental conditions as mentioned above.

Table 1. Chemical composition of essential oil of E. camaldulensis Dehnh.

\begin{tabular}{llcc}
\hline $\mathbf{R I}^{\mathbf{a}}$ & Components & $\mathbf{( \% )}$ & $\begin{array}{c}\text { Identification } \\
\text { methods }\end{array}$ \\
\hline 924 & $\alpha$-Thujene & 0.31 & GC, GC-MS \\
932 & $\alpha$-Pinene & 2.20 & GC, GC-MS \\
1002 & $\alpha$-Phellandrene & 0.51 & GC, GC-MS \\
1020 & $p$-Cymene & 23.95 & GC, GC-MS \\
1026 & 1,8 -Cineole & 32.85 & GC, GC-MS \\
1054 & $\gamma$-Terpinene & 0.64 & GC, GC-MS \\
1183 & Cryptone & 6.79 & GC, GC-MS \\
1238 & Cuminaldehyde & 2.65 & GC, GC-MS \\
1412 & $\beta$-Caryophyllene & 7.63 & GC, GC-MS \\
1439 & Aromadendrene & 1.74 & GC, GC-MS \\
1458 & Alloaromadendrene & 6.05 & GC, GC-MS \\
1496 & Viridiflorene & 1.53 & GC, GC-MS \\
1500 & Bicyclogermacrene & 5.65 & GC, GC-MS \\
1582 & Caryophylleneoxide & 4.03 & GC, GC-MS \\
Monoterpene hydrocarbons $(\%)$ & 27.61 & \\
Oxygenated monoterpenes $(\%)$ & 42.29 & \\
Sesquiterpene hydrocarbons $(\%)$ & 22.60 & \\
Oxygenated sesquiterpenes(\%) & 4.03 & \\
Total & (\%) & 96.53 & \\
\hline
\end{tabular}

${ }^{\text {a}}$ Retention index relative to n-alkanes on SGE-BPX5 capillary column; GC: identification based on retention times of authentic compounds on SGE-BPX5 capillary column; MS, RI: tentatively identified based on computer matching of the masss pectra of peaks with Wiley $7 \mathrm{~N}$ and TRLIB libraries and published data, and comparison of retention index of the compounds compared with published data [22-24].

\subsection{Bioassays}

In order to test the toxicities of E. camaldulensis oil, at 5, 10 and $20 \mu \mathrm{L}$ of essential oil were impregnated into Whatman no. 1 filter paper, which was stuck onto the inner top of the petri dishes where the insects would be placed. This prevented direct contact between the oils and the adult insects. Thirtythree adults of $R$. dominica, $S$. granarius, $C$. maculatus, A. obtectus and $T$. confusum were placed onto filter paper containing adequate amounts of wheat grains, cracked grains, uninfected flour, cowpea seeds and kidney bean seeds. The petri dishes were covered with a lid and transferred to an incubator, and then kept under standard conditions at $25 \pm 2{ }^{\circ} \mathrm{C}, 65 \pm 5 \mathrm{rh}$. and in the darkness for two days. Mortalities of the adults were counted on the $1^{\text {st }}, 2^{\text {nd }}, 3^{\text {rd }}, 4^{\text {th }}$ and the $5^{\text {th }}$ days. For each species, another petri dish treated with only sterile water was used as control. Each assay was repeated three times for each concentration and exposure time combination, and insecticidal activities of the E. camaldulensis oil were expressed as percent mean mortalities of the adult insects.

\subsection{Fungi Material}

The plant pathogenic fungi were obtained from the culture collection at Atatürk University. All fungi cultures were maintained on potato dextrose agar (PDA) and stored at $4^{\circ} \mathrm{C}$. The fungal species used in the experiments were V. dahliae, F. oxyporum, P. debaryanum, S. sclerotiorum and R. solani. Antifungal 
activity was studied by using a contact assay (in vitro), which produces hyphal growth inhibition [4]. Briefly, potato dextrose agar (PDA) plates were prepared in $9 \mathrm{~cm}$ diameter glass petri dishes. The essential oil was dissolved in dimethyl sulfoxide (DMSO) (Merck) at different concentrations $(1 \%, \mathrm{v} / \mathrm{v})(0.25,0.5$ and $1.0 \mathrm{mg} / \mathrm{mL}$ concentration) and required amounts of the solutions $(20.0 \mathrm{mg} / \mathrm{Petri}$ dish) were added to each of the PDA plates containing $20 \mathrm{~mL}$ of agar at $50{ }^{\circ} \mathrm{C}$. A disc $(5 \mathrm{~mm}$ diameter $)$ of the fungal species was cut from 1 week old cultures on PDA plates and then the mycelial surface of the disc was placed upside down on the centre of a dish with fungal species in contact with growth medium on the dish. Then, the plates were incubated in the dark at $25 \pm 2^{\circ} \mathrm{C}$. Extension diameter $(\mathrm{mm})$ of hyphae from centers to the sides of the dishes and stored at $4^{\circ} \mathrm{C}$. The diameter of the fungal species used in the dishes were measured at 24-h intervals for 7 days. Mean of growth measurements were calculated from four replicates of each of the fungal species. PDA plates containing DMSO \pm water solution $(1 \%, \mathrm{v} / \mathrm{v})$, without essential oil solution were used as negative control. In addition, PDA plates treated with captan wp ( $20.0 \mathrm{mg} /$ Petri dish) were used as positive control. Mycelial growth inhibition (GI) was calculated as a percentage from the difference between growth of treated and control mycelium using the following equations:

$$
\text { GI }(\%)=(\mathrm{C}-\mathrm{T} / \mathrm{C}) \times 100
$$

Where, $\mathrm{C}$ is mean of hypal extension $(\mathrm{mm})$ of negative controls and $\mathrm{T}$ is mean of hyphal extension $(\mathrm{mm})$ of plates treated with the tested compounds.

\subsection{Weed Material and Seedling Growth Experiments}

The seeds of $C$. arvensis, $M$. officinalis and A. retroflexus were collected in the Erzurum region (Turkey) in October 2015. Empty and undeveloped seeds were discarded by floating in tap water. To avoid possible inhibition caused by toxins from fungi or bacteria, the seeds were surface sterilized with $15 \%$ sodium hypochlorite for $20 \mathrm{~min}$. and then rinsed with abundant distilled water. Trifluralin (Mega-Tref 48 EC) was used as a positive control. To determine the contact herbicidal effect of the oil, the oil was dissolved in DMSO-water solution $(10 \%, \mathrm{v} / \mathrm{v})$. The emulsions were transferred to Petri dish $(9 \mathrm{~cm}$ diameter) placed on the bottom two layers of filter paper (10 $\mu \mathrm{L} /$ Petri dishes). Afterwards, 50 seeds of $C$. arvensis, M. officinalis and A. retroflexus were placed on the filter paper [7, 25]. Petri dishes were closed with an adhesive tape to prevent escaping of volatile compounds and were kept at $23 \pm 2^{\circ} \mathrm{C}$ on a growth chamber supply with $12 \mathrm{~h}$ of fluorescent light and humidity of $80 \%$ [26]. After 10 days, the number of germinated seeds was determined and stem and root lengths were measured. Germination was measured as the percentage of seeds from which a radicle emerges. The treatments were arranged in a completely randomized design with three replications including controls.

\subsection{Statistical Analysis}

In order to determine whether there is a statistically significant difference among the obtained results for antifungal and herbicidal activity assays, variance analyses were carried out using SPSS 20 software package. Differences between means were tested by Duncan test and values with $(\mathrm{p} \leq 0.05)$ were considered significantly different.

\section{Results and Discussion}

\subsection{The Insecticidal Effects of Essential Oil}

When the toxic effects of $E$. camaldulensis essential oil concentrations and duration were evaluated, the difference between the treated and untreated samples were found to be statistically significant in most cases (Dose $\mathrm{F}_{3,40}=1801.61 ; \mathrm{P}<0.0001$, Day $\mathrm{F}_{4,40}=17.18 ; \mathrm{P}<0.0001$ ). The mortality rates of $R$. dominica adults were found as $60.6 \%$ at $5 \mu \mathrm{L}$, at $25 \pm 2{ }^{\circ} \mathrm{C}$ on the $5^{\text {th }}$ day, while it was measured as $93.9 \%$ at $10 \mu \mathrm{L}$ and $100 \%$ at $20 \mu \mathrm{L}$ on the $1^{\text {st }}$ day (Table 2). The differences of the applications were found statistically significant. 
Table 2. The effect of eucalyptus volatile oil concentrations and treatment durations on the death rate of $R$. dominica adults.

\begin{tabular}{|c|c|c|c|c|c|c|}
\hline \multirow[b]{2}{*}{ Concentrations } & \multicolumn{5}{|c|}{ Mortality rates of $R$. dominica \pm Standard error } & \multirow[b]{2}{*}{$\mathbf{P}$ and $\mathbf{F}$} \\
\hline & $1^{\text {st day }}$ & $2^{\text {nd }}$ day & $3^{\text {rd day }}$ & $4^{\text {th }}$ day & $5^{\text {th }}$ day & \\
\hline \multirow{2}{*}{$5 \mu \mathrm{L}$} & $30.3 \pm 4.6$ & $43.4 \pm 3.6$ & $48.5 \pm 6.3$ & $54.5 \pm 7.6$ & $60.6 \pm 4.6$ & $\mathrm{~F}_{4,10}=4.37$ \\
\hline & $\mathrm{Cb}$ & $\mathrm{Cab}$ & $\mathrm{Ca}$ & $\mathrm{Ca}$ & $\mathrm{Ca}$ & $\mathrm{P}<0.05$ \\
\hline \multirow{2}{*}{$10 \mu \mathrm{L}$} & $80.8 \pm 2.7$ & $86.9 \pm 2.7$ & $89.9 \pm 1.0$ & $91.9 \pm 1.0$ & $93.9 \pm 0$ & $\mathrm{~F}_{4,10}=9.28$ \\
\hline & $\mathrm{Bc}$ & Bbc & $\mathrm{Bab}$ & $\mathrm{Bab}$ & $\mathrm{Ba}$ & $\mathrm{P}<0.01$ \\
\hline \multirow{2}{*}{$20 \mu \mathrm{L}$} & $100 \pm 0$ & $100 \pm 0$ & $100 \pm 0$ & $100 \pm 0$ & $100 \pm 0$ & $\mathrm{~F}_{4,10}=-$ \\
\hline & A & A & A & A & A & $P=-$ \\
\hline \multirow{2}{*}{ Control } & $0 \pm 0$ & $0 \pm 0$ & $0 \pm 0$ & $2 \pm 0$ & $5 \pm 1$ & $\mathrm{~F}_{4,10}=12.67$ \\
\hline & Dc & Dc & Dc & $\mathrm{Db}$ & $\mathrm{Da}$ & $\mathrm{P}<0.001$ \\
\hline \multirow{2}{*}{$\mathrm{P}$ and $\mathrm{F}$} & $\mathrm{F}_{3,8}=483.9$ & $\mathrm{~F}_{3,8}=634.4$ & $F_{3,8}=430.5$ & $\mathrm{~F}_{3,8}=165.7$ & $\mathrm{~F}_{3,8}=491.0$ & \\
\hline & $\mathrm{P}<0.0001$ & $\mathrm{P}<0.0001$ & $\mathrm{P}<0.0001$ & $\mathrm{P}<0.0001$ & $\mathrm{P}<0.0001$ & \\
\hline
\end{tabular}

The mortality rates were recorded $100 \%$ at $5 \mu \mathrm{L}$ concentrations on the $5^{\text {th }}$ day, at $10 \mu \mathrm{L}$ on the $3^{\text {rd }}$ day and at $20 \mu \mathrm{L}$ on the $2^{\text {nd }}$ day for $S$. granarius adults at $25 \pm 2{ }^{\circ} \mathrm{C}$ (Dose $\mathrm{F}_{3,40}=917.36$; $\mathrm{P}<0.0001$, Day $\left.\mathrm{F}_{4,40}=29.36 ; \mathrm{P}<0.0001\right)$. The differences between the applications were determined as statistically significant (Table 3).

Table 3. The effect of eucalyptus volatile oil concentrations and treatment durations on the death rate of $S$. granarius adults.

\begin{tabular}{|c|c|c|c|c|c|c|}
\hline \multicolumn{7}{|c|}{ Mortality rates of $S$. granarius \pm Standard error } \\
\hline Concentrations & $1^{\text {st }}$ day & $2^{\text {nd }}$ day & $3^{\text {rd }}$ day & $4^{\text {th }}$ day & $5^{\text {th }}$ day & $P$ and $F$ \\
\hline \multirow{2}{*}{$5 \mu \mathrm{L}$} & $74.7 \pm 5.0$ & $81.8 \pm 4.6$ & $89.9 \pm 3.6$ & $97 \pm 1.7$ & $100 \pm 0$ & $\mathrm{~F}_{4,10}=12.51$ \\
\hline & $\mathrm{Cd}$ & Bcd & $\mathrm{Bbc}$ & $\mathrm{Bab}$ & $\mathrm{Aa}$ & $\mathrm{P}<0.001$ \\
\hline \multirow{2}{*}{$10 \mu \mathrm{L}$} & $88.8 \pm 4.0$ & $92.9 \pm 3.6$ & $100 \pm 0$ & $100 \pm 0$ & $100 \pm 0$ & $\mathrm{~F}_{4,10}=6.58$ \\
\hline & $\mathrm{Bb}$ & $\mathrm{Bb}$ & $\mathrm{Aa}$ & Aa & Aa & $\mathrm{P}<0.01$ \\
\hline \multirow{2}{*}{$20 \mu \mathrm{L}$} & $98.9 \pm 1.0$ & $100 \pm 0$ & $100 \pm 0$ & $100 \pm 0$ & $100 \pm 0$ & $\mathrm{~F}_{4,10}=1$ \\
\hline & Aa & $\mathrm{Aa}$ & Aa & Aa & Aa & $\mathrm{P}=0.4516$ \\
\hline \multirow{2}{*}{ Control } & $0 \pm 0$ & $4.0 \pm 1.0$ & $6.0 \pm 1.7$ & $8 \pm 1.0$ & $11.1 \pm 1.0$ & $\mathrm{~F}_{4,10}=31.57$ \\
\hline & Dd & $\mathrm{Cc}$ & $\mathrm{Cbc}$ & $\mathrm{Cab}$ & $\mathrm{Ba}$ & $\mathrm{P}<0.0001$ \\
\hline \multirow{2}{*}{$\mathrm{P}$ and $\mathrm{F}$} & $F_{3,8}=137.7$ & $\mathrm{~F}_{3,8}=85.02$ & $F_{3,8}=256.54$ & $F_{3,8}=266.34$ & $F_{3,8}=5611.48$ & \\
\hline & $P<0.0001$ & $\mathrm{P}<0.0001$ & $\mathrm{P}<0.0001$ & $\mathrm{P}<0.0001$ & $\mathrm{P}<0.0001$ & \\
\hline
\end{tabular}

The elimination of $T$. confusum adults, on the other hand, was determined to be $84.8 \%$ at $5 \mu \mathrm{L}$, $95.9 \%$ at $10 \mu \mathrm{L}$, and $100 \%$ at $20 \mu \mathrm{L}$ on the $5^{\text {th }}$ day of the treatment (Dose $\mathrm{F}_{3,40}=917.36 ; \mathrm{P}<0.0001$, Day $\left.\mathrm{F}_{4,40}=29.36 ; \mathrm{P}<0.0001\right)$. The differences between the applications were determined as statistically significant (Table 4).

Table 4. The effect of eucalyptus volatile oil concentrations and treatment durations on the death rate of $T$. confusum adults.

\begin{tabular}{|c|c|c|c|c|c|c|}
\hline \multirow[b]{2}{*}{ Concentrations } & \multicolumn{5}{|c|}{ Mortality rates of $T$. confusum \pm Standard error } & \multirow[b]{2}{*}{$\mathbf{P}$ and $\mathbf{F}$} \\
\hline & $1^{\text {st day }}$ & $2^{\text {nd }}$ day & $3^{\text {rd day }}$ & $4^{\text {th }}$ day & $5^{\text {th }}$ day & \\
\hline \multirow{2}{*}{$5 \mu \mathrm{L}$} & $17.2 \pm 3.6$ & $28.3 \pm 2.0$ & $53.5 \pm 4.4$ & $67.7 \pm 3.6$ & $84.8 \pm 1.7$ & $F_{4,10}=66.97$ \\
\hline & $\mathrm{Ce}$ & $\mathrm{Cd}$ & $\mathrm{Bc}$ & $\mathrm{Cb}$ & $\mathrm{Ca}$ & $\mathrm{P}<0.0001$ \\
\hline \multirow{2}{*}{$10 \mu \mathrm{L}$} & $40.4 \pm 1.0$ & $50.5 \pm 2.0$ & $68.7 \pm 3.5$ & $84.8 \pm 3.5$ & $95.9 \pm 2.7$ & $\mathrm{~F}_{4,10}=36.38$ \\
\hline & $\mathrm{Bd}$ & $\mathrm{Bd}$ & $\mathrm{Bc}$ & $\mathrm{Bb}$ & $\mathrm{Ba}$ & $\mathrm{P}<0.0001$ \\
\hline \multirow{2}{*}{$20 \mu \mathrm{L}$} & $76.8 \pm 4.4$ & $81.8 \pm 3.5$ & $98.9 \pm 1.0$ & $98.9 \pm 1.0$ & $100 \pm 0$ & $F_{4,10}=14.20$ \\
\hline & $\mathrm{Ab}$ & $\mathrm{Ab}$ & Aa & Аа & Аа & $\mathrm{P}<0.0001$ \\
\hline \multirow{2}{*}{ Control } & $0 \pm 0$ & $1 \pm 1$ & $2 \pm 1$ & $2 \pm 1$ & $5.0 \pm 1.0$ & $\mathrm{~F}_{4,10}=3.20$ \\
\hline & $\mathrm{Db}$ & $\mathrm{Db}$ & $\mathrm{Cab}$ & Dab & $\mathrm{Da}$ & $\mathrm{P}=0.0619$ \\
\hline \multirow{2}{*}{$\mathrm{P}$ and $\mathrm{F}$} & $F_{3,8}=155.99$ & $\mathrm{~F}_{3,8}=126.5$ & $\mathrm{~F}_{3,8}=75.28$ & $\mathrm{~F}_{3,8}=131.35$ & $\mathrm{~F}_{3,8}=160.16$ & \\
\hline & $\mathrm{P}<0.0001$ & $\mathrm{P}<0.0001$ & $\mathrm{P}<0.0001$ & $\mathrm{P}<0.0001$ & $\mathrm{P}<0.0001$ & \\
\hline
\end{tabular}

On the other hand, $100 \%$ of C. maculatus adults was determined to be killed by 5 and $10 \mu \mathrm{L}$ of the oil on the $3^{\text {rd }}$ day, and by $20 \mu \mathrm{L}$ on the $1^{\text {st }}$ day of the experiment (Dose $\mathrm{F}_{3,40}=1417.91 ; \mathrm{P}<0.0001$, Day $\left.\mathrm{F}_{4,40}=29.05 ; \mathrm{P}<0.0001\right)$. The differences between the applications were found statistically significant (Table 5). 
Table 5. The effect of eucalyptus volatile oil concentrations and treatment durations on the death rate of $C$. maculatus adults.

\begin{tabular}{|c|c|c|c|c|c|c|}
\hline \multicolumn{7}{|c|}{ Mortality rates of $C$. maculatus \pm Standard error } \\
\hline Concentrations & $1^{\text {st }}$ day & $2^{\text {nd }}$ day & $3^{\text {rd }}$ day & $4^{\text {th }}$ day & $5^{\text {th }}$ day & $P$ and $F$ \\
\hline \multirow{2}{*}{$5 \mu \mathrm{L}$} & $86.9 \pm 2.7$ & $93.4 \pm 3.5$ & $100 \pm 0$ & $100 \pm 0$ & $100 \pm 0$ & $\mathrm{~F}_{4,10}=7.82$ \\
\hline & $\mathrm{Bc}$ & $\mathrm{Abc}$ & $\mathrm{Aab}$ & $\mathrm{Aa}$ & $\mathrm{Aa}$ & $\mathrm{P}<0.01$ \\
\hline \multirow{2}{*}{$10 \mu \mathrm{L}$} & $91.9 \pm 2.7$ & $99 \pm 1.0$ & $100 \pm 0$ & $100 \pm 0$ & $100 \pm 0$ & $\mathrm{~F}_{4,10}=11.59$ \\
\hline & $\mathrm{Bb}$ & $\mathrm{Aa}$ & $\mathrm{Aa}$ & $\mathrm{Aa}$ & $\mathrm{Aa}$ & $\mathrm{P}<0.001$ \\
\hline \multirow{2}{*}{$20 \mu \mathrm{L}$} & $100 \pm 0$ & $100 \pm 0$ & $100 \pm 0$ & $100 \pm 0$ & $100 \pm 0$ & $\mathrm{~F}_{4,10}=-$ \\
\hline & A & A & A & $\mathrm{A}$ & $\mathrm{A}$ & $P=-$ \\
\hline \multirow{2}{*}{ Control } & $1 \pm 1$ & $5 \pm 1$ & $8 \pm 1.0$ & $10.1 \pm 1$ & $14.1 \pm 1$ & $F_{4,10}=15.98$ \\
\hline & $\mathrm{Cb}$ & $\mathrm{Bc}$ & Bab & Bab & $\mathrm{Ba}$ & $\mathrm{P}<0.001$ \\
\hline \multirow{2}{*}{$\mathrm{P}$ and $\mathrm{F}$} & $F_{3,8}=226.96$ & $F_{3,8}=107.02$ & $F_{3,8}=424.83$ & $\mathrm{~F}_{3,8}=5762.3$ & $F_{3,8}=6472.6$ & \\
\hline & $\mathrm{P}<0.0001$ & $\mathrm{P}<0.0001$ & $\mathrm{P}<0.0001$ & $\mathrm{P}<0.0001$ & $\mathrm{P}<0.0001$ & \\
\hline
\end{tabular}

Lastly, $5 \mu \mathrm{L}$ of the oil caused the elimination of $100 \%$ of A. obtectus adults on the $2^{\text {nd }}$ day at $25 \pm 2$ ${ }^{\circ} \mathrm{C}$ while at 10 and $20 \mu \mathrm{L}$, all insects were dead on the $1^{\text {st }}$ day (Dose $\mathrm{F}_{3,40}=3515.92 ; \mathrm{P}<0.0001$, Day $\mathrm{F}_{4,40}=$ 10.49; $\mathrm{P}<0.0001)$ according to control. The differences between the applications were determined as statistically significant (Table 6).

Table 6. The effect of eucalyptus volatile oil concentrations and treatment durations on the death rate of $A$. obtectus adults.

\begin{tabular}{ccccccc}
\hline & & \multicolumn{2}{c}{ Mortality rates of $\boldsymbol{A}$ obtectus \pm Standard error } & & \\
Concentrations & $\mathbf{1}^{\text {st }} \mathbf{d a y}$ & $\mathbf{2}^{\text {nd }} \mathbf{d a y}$ & $\mathbf{3}^{\text {rd }} \mathbf{d a y}$ & $\mathbf{4}^{\text {th }}$ day & $\mathbf{5}^{\text {th }} \mathbf{d a y}$ & $\mathbf{P}$ and F \\
\hline \multirow{2}{*}{$5 \mu \mathrm{L}$} & $98 \pm 2.0$ & $100 \pm 0$ & $100 \pm 0$ & $100 \pm 0$ & $100 \pm 0$ & $\mathrm{~F} 4,10=1$ \\
& $\mathrm{Aa}$ & $\mathrm{Aa}$ & $\mathrm{Aa}$ & $\mathrm{Aa}$ & $\mathrm{Aa}$ & $\mathrm{P}=0.4516$ \\
$10 \mu \mathrm{L}$ & $100 \pm 0$ & $100 \pm 0$ & $100 \pm 0$ & $100 \pm 0$ & $100 \pm 0$ & $\mathrm{~F}_{4,10}=-$ \\
& $\mathrm{A}$ & $\mathrm{A}$ & $\mathrm{A}$ & $\mathrm{A}$ & $\mathrm{A}$ & $\mathrm{P}=-$ \\
$20 \mu \mathrm{L}$ & $100 \pm 0$ & $100 \pm 0$ & $100 \pm 0$ & $100 \pm 0$ & $100 \pm 0$ & $\mathrm{~F}_{4,10}=-$ \\
& $\mathrm{A}$ & $\mathrm{A}$ & $\mathrm{A}$ & $\mathrm{A}$ & $\mathrm{A}$ & $\mathrm{P}=-$ \\
Control & $2 \pm 1$ & $7 \pm 1$ & $10.1 \pm 1$ & $14.1 \pm 1$ & $17.2 \pm 1$ & $\mathrm{~F}_{4,10}=16.45$ \\
& $\mathrm{Bd}$ & $\mathrm{Bc}$ & $\mathrm{Bbc}$ & $\mathrm{Bba}$ & $\mathrm{Ba}$ & $\mathrm{P}<0.001$ \\
$\mathrm{P}$ and $\mathrm{F}$ & $\mathrm{F}_{3,8}=198.62$ & $\mathrm{~F}_{3,8}=4614.9$ & $\mathrm{~F}_{3,8}=5762.3$ & $\mathrm{~F}_{3,8}=6472.6$ & $\mathrm{~F}_{3,8}=7110.8$ & \\
& $\mathrm{P}<0.0001$ & $\mathrm{P}<0.0001$ & $\mathrm{P}<0.0001$ & $\mathrm{P}<0.0001$ & $\mathrm{P}<0.0001$ & \\
\hline
\end{tabular}

\subsection{The Fungicidal Effects of Essential Oil}

The effect of eucalyptus essential oil whose effect was investigated at various concentrations $(5,10$ and $20 \mu \mathrm{L}$ ) and days on fungal mycelial growth, was found being ineffective on mycelial growth of $V$. dahliae at $5 \mu \mathrm{L}$ concentration up to the $3^{\text {rd }}$ day even though it was found to be effective from the first day to the last (1-7) at 10 and $20 \mu \mathrm{L}$ (Dose $\mathrm{F}_{4,70}=759.95 ; \mathrm{p}<0.0001$, Day $\mathrm{F}_{6,70}=78.42 ; \mathrm{p}<0.0001$ ) (Table 7).

Table 7. The effect of eucalyptus essential oil concentrations and treatment durations on mycelial growth of $V$. dahliae

\begin{tabular}{|c|c|c|c|c|c|c|c|c|}
\hline \multirow[b]{2}{*}{ Conc. } & \multicolumn{6}{|c|}{ Verticillium dahliae Kleb. } & \multirow[b]{2}{*}{$7^{\text {th }}$ day } & \multirow[b]{2}{*}{$\mathrm{P}$ and $\mathrm{F}$} \\
\hline & $1^{\text {st day }}$ & $2^{\text {nd }}$ day & $3^{\text {rd }}$ day & $4^{\text {th }}$ day & $5^{\text {th }}$ day & $6^{\text {th }}$ day & & \\
\hline \multirow{2}{*}{$5 \mu \mathrm{L}$} & $0.7 \pm 0.2$ & $1.4 \pm .2$ & $1.9 \pm 0.3$ & $1.9 \pm 0.3$ & $2.7 \pm 0.3$ & $4.6 \pm 0.5$ & $6.9 \pm 1.2$ & $\mathrm{~F}_{6,14}=17.17$ \\
\hline & $\mathrm{Cd}$ & Bdc & Adc & Bdc & $\mathrm{Bc}$ & $\mathrm{Bb}$ & $\mathrm{Ba}$ & $\mathrm{P}<0.0001$ \\
\hline \multirow{2}{*}{$10 \mu \mathrm{L}$} & $0.5 \pm 0$ & $0.5 \pm 0$ & $0.5 \pm 0$ & $0.5 \pm 0$ & $0.5 \pm 0$ & $0.5 \pm 0$ & $0.5 \pm 0$ & $\mathrm{~F}_{6,14}=-$ \\
\hline & $\mathrm{Ca}$ & $\mathrm{Da}$ & $\mathrm{Ca}$ & $\mathrm{Da}$ & $\mathrm{Ca}$ & $\mathrm{Ca}$ & $\mathrm{Ca}$ & $P=-$ \\
\hline \multirow{2}{*}{$20 \mu \mathrm{L}$} & $0.5 \pm 0$ & $0.5 \pm 0$ & $0.5 \pm 0$ & $0.5 \pm 0$ & $0.5 \pm 0$ & $0.5 \pm 0$ & $0.5 \pm 0$ & $\mathrm{~F}_{6,14}=-$ \\
\hline & $\mathrm{Ca}$ & $\mathrm{Da}$ & $\mathrm{Ca}$ & $\mathrm{Da}$ & $\mathrm{Ca}$ & $\mathrm{Ca}$ & $\mathrm{Ca}$ & $P=-$ \\
\hline \multirow{2}{*}{ Control } & $2.3 \pm 0.01$ & $3.75 \pm 0.02$ & $5 \pm 0.05$ & $6.5 \pm 0.01$ & $7.9 \pm 0.03$ & $9 \pm 0$ & $9 \pm 0$ & $F_{6,14}=8206.5$ \\
\hline & Af & $\mathrm{Ae}$ & Ad & Ac & $\mathrm{Ab}$ & $\mathrm{Aa}$ & $\mathrm{Aa}$ & $\mathrm{P}<0.0001$ \\
\hline \multirow{2}{*}{$\begin{array}{l}\text { Positive } \\
\text { control }\end{array}$} & $1.0 \pm 0.01$ & $1.0 \pm 0.01$ & $1.0 \pm 0.01$ & $1.0 \pm 0.01$ & $1.0 \pm 0.01$ & $1.0 \pm 0.01$ & $1.0 \pm 0.01$ & $\mathrm{~F}_{6,14}=1.03$ \\
\hline & $\mathrm{Ba}$ & $\mathrm{Ca}$ & $\mathrm{Ba}$ & $\mathrm{Da}$ & $\mathrm{Ca}$ & $\mathrm{Ca}$ & $\mathrm{Ca}$ & $\mathrm{P}=0.4488$ \\
\hline \multirow{2}{*}{$\mathrm{P}$ and $\mathrm{F}$} & $\mathrm{F}_{4,10}=55.40$ & $F_{4,10}=237.47$ & $F_{4,10}=234.37$ & $F_{4,10}=8633.8$ & $F_{4,10}=632.52$ & $F_{4,10}=286.3$ & $\mathrm{~F}_{4,10}=60.8$ & \\
\hline & $\mathrm{P}<0.0001$ & $\mathrm{P}<0.0001$ & $\mathrm{P}<0.0001$ & $\mathrm{P}<0.0001$ & $\mathrm{P}<0.0001$ & $\mathrm{P}<0.0001$ & $\mathrm{P}<0.0001$ & \\
\hline
\end{tabular}

ANOVA was applied to the data, and the differences between the mean values were given at the $5 \%$ significance level according to DUNCAN test. 
Positive control was also found to be effective in 7 days. Eucalyptus essential oil was found to be effective on mycelial growth of $P$. debaryanum at 5,10 and $20 \mu \mathrm{L}$ in the first 5 days but it was found ineffective at $5 \mu \mathrm{L}$, on the $6^{\text {th }}$ and $7^{\text {th }}$ days according to control (Dose $F_{4,40}=420.88 ; p<0.0001$, Day $\left.\mathrm{F} 6{ }_{, 40}=182.41 ; \mathrm{p}<0.0001\right)$. The difference was significant at 10 and $20 \mu \mathrm{L}$ concentration in 7 days according to control (Table 8).

Table 8. The effect of eucalyptus essential oil concentrations and treatment durations on mycelial growth of $P$. debaryanum.

\begin{tabular}{|c|c|c|c|c|c|c|c|c|}
\hline \multicolumn{9}{|c|}{ Phytium debaryanum Auct. non R. Hesse } \\
\hline Conc. & $1^{\text {st }}$ day & $2^{\text {nd }}$ day & $3^{\text {rd day }}$ & $4^{\text {th }}$ day & $5^{\text {th }}$ day & $6^{\text {th }}$ day & $7^{\text {th }}$ day & $\mathrm{P}$ and $\mathrm{F}$ \\
\hline \multirow{2}{*}{$5 \mu \mathrm{L}$} & $2.4 \pm 0.07$ & $3.5 \pm 0.02$ & $4.2 \pm 0.2$ & $6.4 \pm 0.2$ & $7.4 \pm 0.3$ & $8.3 \pm 0.3$ & $9 \pm 0$ & $\mathrm{~F}_{6,14}=128.93$ \\
\hline & $\mathrm{Bf}$ & $\mathrm{Be}$ & $\mathrm{Bd}$ & $\mathrm{Bc}$ & $\mathrm{Bb}$ & $\mathrm{Aa}$ & $\mathrm{Aa}$ & $\mathrm{P}<0.0001$ \\
\hline \multirow{2}{*}{$10 \mu \mathrm{L}$} & $1.5 \pm 0.08$ & $2.05 \pm 0.18$ & $2.7 \pm 0.2$ & $3.7 \pm 0.3$ & $4.9 \pm 0.2$ & $5.9 \pm 0.2$ & $7.1 \pm 0.2$ & $F_{6,14}=113.96$ \\
\hline & Df & $\mathrm{Cf}$ & De & $\mathrm{Cd}$ & $\mathrm{Cc}$ & $\mathrm{Bb}$ & $\mathrm{Ba}$ & $\mathrm{P}<0.0001$ \\
\hline \multirow{2}{*}{$20 \mu \mathrm{L}$} & $1.2 \pm 0.08$ & $1.5 \pm 0.18$ & $1.9 \pm 0.3$ & $2.3 \pm 0.4$ & $2.7 \pm 0.6$ & $3.3 \pm 0.8$ & $3.9 \pm 0.8$ & $\mathrm{~F}_{6,14}=3.57$ \\
\hline & $\mathrm{Ec}$ & $\mathrm{Dc}$ & Ecb & Dbac & Dbac & $\mathrm{Cba}$ & $\mathrm{Ca}$ & $\mathrm{P}<0.05$ \\
\hline \multirow{2}{*}{ Control } & $4.5 \pm 0.08$ & $6.5 \pm 0.05$ & $7.7 \pm 0.14$ & $9 \pm 0$ & $9 \pm 0$ & $9 \pm 0$ & $9 \pm 0$ & $\mathrm{~F}_{6,14}=661.73$ \\
\hline & Ad & Ac & $\mathrm{Ab}$ & $\mathrm{Aa}$ & $\mathrm{Aa}$ & $\mathrm{Aa}$ & $\mathrm{Aa}$ & $\mathrm{P}<0.0001$ \\
\hline \multirow{2}{*}{$\begin{array}{l}\text { Positive } \\
\text { control }\end{array}$} & $1.8 \pm 0.04$ & $2.6 \pm 0.1$ & $3.5 \pm .1$ & $4.1 \pm .1$ & $4.4 \pm 0.1$ & $4.9 \pm 0.1$ & $4.9 \pm .1$ & $F_{6,14}=86.52$ \\
\hline & $\mathrm{Ce}$ & $\mathrm{Cd}$ & $\mathrm{Cc}$ & $\mathrm{Cb}$ & $\mathrm{Cb}$ & $\mathrm{Ba}$ & $\mathrm{Ca}$ & $\mathrm{P}<0.0001$ \\
\hline $\mathrm{P}$ and $\mathrm{F}$ & $\mathrm{F}_{4,10}=304.29$ & $\begin{array}{c}\mathrm{F}_{4,10}=147.53 \\
\mathrm{P}<0.0001\end{array}$ & $\begin{array}{c}\mathrm{F}_{4,10}=117.11 \\
\mathrm{P}<0.0001\end{array}$ & $\begin{array}{c}\mathrm{F}_{4,10}=117.83 \\
\mathrm{P}<0.0001\end{array}$ & $\begin{array}{l}\mathrm{F}_{4,10}=60.24 \\
\mathrm{P}<0.0001\end{array}$ & $\begin{array}{c}\mathrm{F}_{4,10}=38.84 \\
\mathrm{P}<0.0001\end{array}$ & $\mathrm{~F}_{4,10}=38.47$ & \\
\hline
\end{tabular}

ANOVA was applied to the data, and the differences between the mean values were given at the 5\% significance le vel according to DUNCAN test.

The result also showed that the effect of eucalyptus essential oil was significantly on mycelial growth of $F$. oxyporum at 5,10 and $20 \mu \mathrm{L}$ concentration in 7 days compared to the control (Dose $\mathrm{F} 4,40=$ 1735.06; $<<0.0001$, Day F6, $40=546.34 ; \mathrm{p}<0.0001)($ Table 9).

Table 9. The effect of eucalyptus essential oil concentrations and treatment durations on mycelial growth of $F$. oxyporum

\begin{tabular}{|c|c|c|c|c|c|c|c|c|}
\hline \multicolumn{9}{|c|}{ Fusarium oxyporum Schl. } \\
\hline Conc. & $\mathbf{1}^{\text {st day }}$ & $2^{\text {nd }}$ day & $3^{\text {rd }}$ day & $4^{\text {th }}$ day & $5^{\text {th }}$ day & $6^{\text {th }}$ day & $7^{\text {th }}$ day & $\mathrm{P}$ and $\mathrm{F}$ \\
\hline \multirow{2}{*}{$5 \mu \mathrm{L}$} & $1.6 \pm 0.07$ & $2.2 \pm 0.07$ & $2.5 \pm 0.08$ & $3.3 \pm 0.11$ & $3.8 \pm 0.15$ & $4.5 \pm 0.14$ & $5.1 \pm 0.18$ & $\mathrm{~F}_{6,14=102.45}$ \\
\hline & $\mathrm{Bf}$ & $\mathrm{Be}$ & $\mathrm{Be}$ & $\mathrm{Bd}$ & $\mathrm{Bc}$ & $\mathrm{Bb}$ & $\mathrm{Ba}$ & $\mathrm{P}<0.0001$ \\
\hline \multirow{2}{*}{$10 \mu \mathrm{L}$} & $1.4 \pm 0.02$ & $1.8 \pm 0.1$ & $2.2 \pm 0.07$ & $2.6 \pm 0.1$ & $2.9 \pm .06$ & $3.4 \pm 0.03$ & $3.75 \pm 0.1$ & $\mathrm{~F}_{6,14}=122.23$ \\
\hline & $\mathrm{Cg}$ & $\mathrm{Cf}$ & $\mathrm{Ce}$ & $\mathrm{Cd}$ & $\mathrm{Cc}$ & $\mathrm{Cb}$ & $\mathrm{Ca}$ & $\mathrm{P}<0.0001$ \\
\hline \multirow{2}{*}{$20 \mu \mathrm{L}$} & $1.1 \pm 0.05$ & $1.4 \pm 0.06$ & $1.8 \pm 0.09$ & $2.0 \pm 0.1$ & $2.3 \pm 0.1$ & $2.6 \pm .1$ & $2.8 \pm 0.15$ & $\mathrm{~F}_{6,14}=33.28$ \\
\hline & $\mathrm{De}$ & $\mathrm{Dd}$ & Dc & Dcb & $\mathrm{Db}$ & Da & $\mathrm{Da}$ & $\mathrm{P}<0.0001$ \\
\hline \multirow{2}{*}{ Control } & $2.3 \pm 0.01$ & $3.3 \pm 0.01$ & $4.1 \pm 0.1$ & $5.2 \pm 0.04$ & $5.9 \pm 0.0$ & $7.1 \pm 0.07$ & $8.2 \pm 0.05$ & $F_{6,14}=356.39$ \\
\hline & $\mathrm{Ag}$ & Af & $\mathrm{Ae}$ & Ad & Ac & $\mathrm{Ab}$ & $\mathrm{Aa}$ & $\mathrm{P}<0.0001$ \\
\hline \multirow{2}{*}{$\begin{array}{l}\text { Positive } \\
\text { control }\end{array}$} & $0.5 \pm 0$ & $0.5 \pm 0$ & $1.0 \pm 0.01$ & $1.1 \pm 0.04$ & $1.3 \pm 0.08$ & $1.3 \pm 0.08$ & $1.3 \pm 0.08$ & $F_{6,14}=41.18$ \\
\hline & Ac & $\mathrm{Ec}$ & $\mathrm{Eb}$ & $\mathrm{Eb}$ & $\mathrm{Ea}$ & $\mathrm{Ea}$ & $\mathrm{Ea}$ & $\mathrm{P}<0.0001$ \\
\hline \multirow{2}{*}{$\mathrm{P}$ and $\mathrm{F}$} & $F_{4,10}=220.76$ & $\mathrm{~F}_{4,10}=158.53$ & $F_{4,10}=166.53$ & $\mathrm{~F}_{4,10}=266.41$ & $\mathrm{~F}_{4,10}=171.28$ & $F_{4,10}=490.92$ & $\mathrm{~F}_{4,10}=438.83$ & \\
\hline & $\mathrm{P}<0.0001$ & $\mathrm{P}<0.0001$ & $\mathrm{P}<0.0001$ & $\mathrm{P}<0.0001$ & $\mathrm{P}<0.0001$ & $\mathrm{P}<0.0001$ & $\mathrm{P}<0.0001$ & \\
\hline
\end{tabular}

ANOVA was applied to the data, and the differences between the mean values were given at the 5\% significance level according to DUNCAN test.

The effect of eucalyptus essential oil was significant on mycelial growth of S. sclerotiorum at 10 and $20 \mu \mathrm{L}$ concentrations in first 4 days (Dose F4, ${ }_{40}=3298.17$; $\mathrm{p}<0.0001$, Day $\mathrm{F}_{, 40}=1152.53$; $\mathrm{p}<0.0001$ ), whereas it was insignificant at $5 \mu \mathrm{L}$ in 5-7 days (Table 10). But it was found significant at 20 $\mu \mathrm{L}$ in 1-7 days. 
Table 10. The effect of eucalyptus essential oil concentrations and treatment durations on mycelial growth of S. sclerotiorum

\begin{tabular}{|c|c|c|c|c|c|c|c|c|}
\hline \multirow[b]{2}{*}{ Conc. } & \multirow[b]{2}{*}{$\mathbf{1}^{\text {st day }}$} & \multicolumn{6}{|c|}{ Sclerotinia sclerotiorum (Lib.) de Barry } & \multirow[b]{2}{*}{$\mathrm{P}$ and $\mathrm{F}$} \\
\hline & & $2^{\text {nd }}$ day & $3^{\text {rd }}$ day & $4^{\text {th }}$ day & $5^{\text {th }}$ day & $6^{\text {th }}$ day & $7^{\text {th }}$ day & \\
\hline \multirow{2}{*}{$5 \mu \mathrm{L}$} & $0.5 \pm 0$ & $1.4 \pm 0.06$ & $3.8 \pm 1.3$ & $6.8 \pm 0.2$ & $9 \pm 0$ & $9 \pm 0$ & $9 \pm 0$ & $F_{6,14}=1819.6$ \\
\hline & $\mathrm{Be}$ & $\mathrm{Bd}$ & $\mathrm{Bc}$ & $\mathrm{Bb}$ & $\mathrm{Aa}$ & $\mathrm{Aa}$ & Aa & $\mathrm{P}<0.0001$ \\
\hline \multirow{2}{*}{$10 \mu \mathrm{L}$} & $0.5 \pm 0$ & $1.0 \pm 0.01$ & $1.3 \pm 0.1$ & $3.0 \pm 0.2$ & $5.2 \pm 0.4$ & $7.5 \pm 0.4$ & $9 \pm 0$ & $\mathrm{~F}_{6,14}=194.51$ \\
\hline & $\mathrm{Be}$ & $\mathrm{Ce}$ & $\mathrm{Ce}$ & $\mathrm{Cd}$ & $\mathrm{Bc}$ & $\mathrm{Bb}$ & Aa & $\mathrm{P}<0.0001$ \\
\hline \multirow{2}{*}{$20 \mu \mathrm{L}$} & $0.5 \pm 0$ & $0.5 \pm 0$ & $0.5 \pm 0$ & $1.0 \pm 0.03$ & $1.6 \pm 0.08$ & $2.4 \pm 0.1$ & $3.6 \pm 0.1$ & $F_{6,14}=273.47$ \\
\hline & $\mathrm{Be}$ & $\mathrm{De}$ & De & $\mathrm{Dd}$ & $\mathrm{Cc}$ & $\mathrm{Cb}$ & $\mathrm{Ba}$ & $\mathrm{P}<0.0001$ \\
\hline \multirow{2}{*}{ Control } & $2.9 \pm 0.2$ & $5.4 \pm 0.2$ & $7.9 \pm 0.2$ & $9 \pm 0$ & $9 \pm 0$ & $9 \pm 0$ & $9 \pm 0$ & $F_{6,14}=353.27$ \\
\hline & Ad & $\mathrm{Ac}$ & $\mathrm{Ab}$ & $\mathrm{Aa}$ & $\mathrm{Aa}$ & $\mathrm{Aa}$ & $\mathrm{Aa}$ & $\mathrm{P}<0.0001$ \\
\hline \multirow{2}{*}{$\begin{array}{l}\text { Positive } \\
\text { control }\end{array}$} & $0.5 \pm 0$ & $0.5 \pm 0$ & $0.5 \pm 0$ & $0.5 \pm 0$ & $0.5 \pm 0$ & $0.5 \pm 0$ & $0.5 \pm 0$ & $F_{6,14}=-$ \\
\hline & $\mathrm{Ba}$ & $\mathrm{Da}$ & $\mathrm{Da}$ & $\mathrm{Ea}$ & $\mathrm{Da}$ & $\mathrm{Da}$ & $\mathrm{Ca}$ & $P=-$ \\
\hline $\mathrm{P}$ and $\mathrm{F}$ & $\begin{array}{c}\mathrm{F}_{4,10}=167.51 \\
\mathrm{P}<0.0001\end{array}$ & $\begin{array}{c}\mathrm{F}_{4,10}= \\
539.04 \\
\mathrm{P}<0.0001\end{array}$ & $\begin{array}{c}\mathrm{F}_{4,10}= \\
601.63 \\
\mathrm{P}<0.0001\end{array}$ & $\begin{array}{c}\mathrm{F}_{4,10}=973.95 \\
\mathrm{P}<0.0001\end{array}$ & $\begin{array}{c}\mathrm{F}_{4,10}=384.18 \\
\mathrm{P}<0.0001\end{array}$ & $\begin{array}{c}\mathrm{F}_{4,10}=486.33 \\
\mathrm{P}<0.0001\end{array}$ & $\begin{array}{c}\mathrm{F}_{4,10}=4464.86 \\
\mathrm{P}<0.0001\end{array}$ & \\
\hline
\end{tabular}

ANOVA was applied to the data, and the differences between the mean values were given at the 5\% significance level according to DUNCAN test.

The result showed that eucalyptus essential oil was found to be ineffective on mycelial growth of $R$. solani at 5, 10 and $20 \mu \mathrm{L}$ concentrations in 7 days $\left(\right.$ Dose $\mathrm{F}_{3,40}=23.24 ; \mathrm{p}<0.0001$, Day $\mathrm{F}_{4,40}=7.70$; $\mathrm{p}<0.0001)($ Table 11).

Table 11. The effect of eucalyptus essential oil concentrations and treatment durations on mycelial growth of $R$. solani.

\begin{tabular}{|c|c|c|c|c|c|c|c|c|}
\hline \multicolumn{9}{|c|}{ Rhizoctania solani Kühn } \\
\hline & $1^{\text {st }}$ day & $2^{\text {nd }}$ day & $3^{\text {rd }}$ day & $4^{\text {th }}$ day & $5^{\text {th }}$ day & $6^{\text {th }}$ day & $7^{\text {th }}$ day & $\mathrm{P}$ and $\mathrm{F}$ \\
\hline $5 \mu \mathrm{L}$ & $\begin{array}{c}1.3 \pm 0.1 \\
\mathrm{Bd}\end{array}$ & $\begin{array}{c}1.5 \pm 0.08 \\
\mathrm{Cdc}\end{array}$ & $\begin{array}{c}1.6 \pm 0.1 \\
\mathrm{Cdcb}\end{array}$ & $\begin{array}{c}1.7 \pm 0.1 \\
\text { Babcd }\end{array}$ & $\begin{array}{c}1.9 \pm 0.2 \\
\text { Babc }\end{array}$ & $\begin{array}{c}2.1 \pm 0.3 \\
\text { Bab }\end{array}$ & $\begin{array}{c}3.3 \pm 0.1 \\
\mathrm{ABa}\end{array}$ & $\begin{array}{c}\mathrm{F}_{6,14=} \\
3.77 \\
\mathrm{P}<0.05\end{array}$ \\
\hline $10 \mu \mathrm{L}$ & $\begin{array}{c}1.7 \pm 0.2 \\
\mathrm{Bb}\end{array}$ & $\begin{array}{c}2.1 \pm 0.4 \\
\mathrm{BCab}\end{array}$ & $\begin{array}{c}2.1 \pm 0.3 \\
\text { BCab }\end{array}$ & $\begin{array}{c}2.4 \pm 0.3 \\
\mathrm{ABab}\end{array}$ & $\begin{array}{c}2.6 \pm 0.2 \\
\mathrm{ABa}\end{array}$ & $\begin{array}{c}2.8 \pm 0.2 \\
\mathrm{ABa}\end{array}$ & $\begin{array}{c}2.8 \pm 0.1 \\
\mathrm{ABa}\end{array}$ & $\begin{array}{c}\mathrm{F}_{6,14}=3.02 \\
\mathrm{P}<0.05\end{array}$ \\
\hline $20 \mu \mathrm{L}$ & $\begin{array}{c}1.6 \pm 0.07 \\
\mathrm{Bb}\end{array}$ & $\begin{array}{c}1.85 \pm 0.20 \\
\mathrm{BCab}\end{array}$ & $\begin{array}{c}2.1 \pm 0.2 \\
\text { BCab }\end{array}$ & $\begin{array}{c}2.3 \pm 0.3 \\
\mathrm{Bab}\end{array}$ & $\begin{array}{c}2.55 \pm 0.3 \\
\mathrm{ABa}\end{array}$ & $\begin{array}{c}2.55 \pm 0.3 \\
\mathrm{ABa}\end{array}$ & $\begin{array}{c}2.55 \pm 0.3 \\
\mathrm{ABa}\end{array}$ & $\begin{array}{c}\mathrm{F}_{6,14}=2.50 \\
\mathrm{P}=0.0744\end{array}$ \\
\hline Control & $\begin{array}{c}2 \pm 0.05 \\
\mathrm{ABe}\end{array}$ & $\begin{array}{c}2.4 \pm 0.05 \\
\mathrm{ABd}\end{array}$ & $\begin{array}{c}2.6 \pm 0.07 \\
\mathrm{ABcd}\end{array}$ & $\begin{array}{c}2.7 \pm 0.08 \\
\mathrm{ABc}\end{array}$ & $\begin{array}{c}2.8 \pm 0.08 \\
\mathrm{ABbc}\end{array}$ & $\begin{array}{c}3 \pm 0.07 \\
\mathrm{ABab}\end{array}$ & $\begin{array}{c}3.1 \pm 0.1 \\
\mathrm{ABa}\end{array}$ & $\begin{array}{c}\mathrm{F}_{6,14}= \\
22.42 \\
\mathrm{P}<0.0001\end{array}$ \\
\hline $\begin{array}{l}\text { Positive } \\
\text { control }\end{array}$ & $\begin{array}{c}2.70 .6 \\
\mathrm{Aa}\end{array}$ & $\begin{array}{c}2.9 \pm 0.3 \\
\mathrm{Aa}\end{array}$ & $\begin{array}{c}3.2 \pm 0.5 \\
\mathrm{Aa}\end{array}$ & $\begin{array}{c}3.3 \pm 0.5 \\
\mathrm{Aa}\end{array}$ & $\begin{array}{c}3.4 \pm 0.5 \\
\mathrm{Aa}\end{array}$ & $\begin{array}{c}3.4 \pm 0.5 \\
\mathrm{Aa}\end{array}$ & $\begin{array}{c}3.4 \pm 0.5 \\
\mathrm{Aa}\end{array}$ & $\begin{array}{c}F_{6,14}=0.33 \\
P=0.9096\end{array}$ \\
\hline $\mathrm{P}$ and $\mathrm{F}$ & $\begin{array}{c}\mathrm{F}_{4,10}=3.45 \\
\mathrm{P}=0.0512\end{array}$ & $\begin{array}{c}\mathrm{F}_{4,10}=5.67 \\
\mathrm{P}<0.05\end{array}$ & $\begin{array}{c}\mathrm{F}_{4,10}=4.27 \\
\mathrm{P}<0.005\end{array}$ & $\begin{array}{c}\mathrm{F}_{4,10}=3.83 \\
\mathrm{P}<0.05\end{array}$ & $\begin{array}{l}\mathrm{F}_{4,10}=3.15 \\
\mathrm{P}=0.0642\end{array}$ & $\begin{array}{c}\mathrm{F}_{4,10}=2.58 \\
\mathrm{P}=0.1018\end{array}$ & $\begin{array}{l}\mathrm{F}_{4,10}=2.14 \\
\mathrm{P}=0.1507\end{array}$ & \\
\hline
\end{tabular}

ANOVA was applied to the data, and the differences between the mean values were given at the $5 \%$ significance level according to DUNCAN test.

\subsection{The Herbicidal Effects of Essential Oil}

Eucalyptus essential oil ineffective on root growth of $C$. arvensis at 5, 10 and $20 \mu \mathrm{L}$ concentrations in 7 days (Table 12), while it was effective on root growth of M. officinalis and A. retroflexus at the same concentrations in 7 days (Dose: $\mathrm{F}_{4,2235}=260.22 \mathrm{P}<0.0001$ ). 
Table 12. The effect of eucalyptus essential oil concentrations and treatment durations on root growth of $C$. arvensis, M. officinalis and A.retroflexus

\begin{tabular}{|c|c|c|c|c|}
\hline Root (cm) & C. arvensis & M. officinalis & A.retroflexus & $P$ and $F$ \\
\hline $5 \mu \mathrm{L}$ & $\begin{array}{c}0.64 \pm 0.09 \\
\mathrm{Ba}\end{array}$ & $\begin{array}{l}0 \pm 0 \\
\mathrm{Cb}\end{array}$ & $\begin{array}{l}0 \pm 0 \\
\mathrm{Bb}\end{array}$ & $\begin{array}{c}\mathrm{F}_{2,447}=42.58 \\
\mathrm{P}<0.0001\end{array}$ \\
\hline $10 \mu \mathrm{L}$ & $\begin{array}{c}0.11 \pm 0.02 \\
\mathrm{Da}\end{array}$ & $\begin{array}{l}0 \pm 0 \\
\mathrm{Cb}\end{array}$ & $\begin{array}{l}0 \pm 0 \\
\mathrm{Bb}\end{array}$ & $\begin{array}{c}\mathrm{F}_{2,447}=14.32 \\
\mathrm{P}<0.0001\end{array}$ \\
\hline $20 \mu \mathrm{L}$ & $\begin{array}{c}0.03 \pm 0.01 \\
\mathrm{Da}\end{array}$ & $\begin{array}{l}0 \pm 0 \\
\mathrm{Cb}\end{array}$ & $\begin{array}{c}0 \pm 0 \\
\mathrm{Bb}\end{array}$ & $\begin{array}{c}\mathrm{F}_{2,447}=6.19 \\
\mathrm{P}<0.0001\end{array}$ \\
\hline Control & $\begin{array}{c}1.58 \pm 0.14 \\
\mathrm{Aa}\end{array}$ & $\begin{array}{c}1.61 \pm 0.12 \\
\mathrm{Ab}\end{array}$ & $\begin{array}{c}0.83 \pm 0.07 \\
\mathrm{Ab}\end{array}$ & $\begin{array}{c}\mathrm{F}_{2,447}=18.57 \\
\mathrm{P}<0.0001\end{array}$ \\
\hline Positive control & $\begin{array}{c}0.36 \pm 0.03 \\
\mathrm{Ca}\end{array}$ & $\begin{array}{c}0.19 \pm 0.03 \\
\mathrm{Bb}\end{array}$ & $\begin{array}{c}0.06 \pm 0.01 \\
\mathrm{Bc}\end{array}$ & $\begin{array}{c}\mathrm{F}_{2,447}=31.22 \\
\mathrm{P}<0.0001\end{array}$ \\
\hline $\mathrm{P}$ and $\mathrm{F}$ & $\begin{array}{c}\mathrm{F}_{4,475}=60.12 \\
\mathrm{P}<0.0001\end{array}$ & $\begin{array}{c}\mathrm{F}_{4,475}=156.84 \\
\mathrm{P}<0.0001\end{array}$ & $\begin{array}{c}\mathrm{F}_{4,475}=134.10 \\
\mathrm{P}<0.0001\end{array}$ & \\
\hline
\end{tabular}

Eucalyptus essential oil was determined as ineffective on stem growth of $C$. arvensis at 5, 10 and $20 \mu \mathrm{L}$ concentrations in 7 days (Table 13), while it was effective on stem growth of $M$. officinalis and $A$. retroflexus at the same concentrations and treatment length (Dose: $\mathrm{F}_{4,2235}=234.27, \mathrm{P}<0.0001$ ).

Table 13. The effect of eucalyptus essential oil concentrations and treatment durations on stem growth of $C$. arvensis, M. officinalis and A.retroflexus

\begin{tabular}{|c|c|c|c|c|}
\hline Stem (cm) & C. arvensis & M. officinalis & A.retroflexus & $P$ and $F$ \\
\hline $5 \mu \mathrm{L}$ & $\begin{array}{c}0.24 \pm 0.04 \\
\mathrm{Ba}\end{array}$ & $\begin{array}{l}0 \pm 0 \\
\mathrm{Bb}\end{array}$ & $\begin{array}{l}0 \pm 0 \\
\mathrm{Bb}\end{array}$ & $\begin{array}{c}\mathrm{F}_{2,447}=25.78 \\
\mathrm{P}<0.0001\end{array}$ \\
\hline $10 \mu \mathrm{L}$ & $\begin{array}{c}0.03 \pm 0.01 \\
\mathrm{Ca}\end{array}$ & $\begin{array}{c}0 \pm 0 \\
\mathrm{Ba}\end{array}$ & $\begin{array}{c}0 \pm 0 \\
\mathrm{Ba}\end{array}$ & $\begin{array}{c}\mathrm{F}_{2,447}=3.12 \\
\mathrm{P}<0.05\end{array}$ \\
\hline $20 \mu \mathrm{L}$ & $\begin{array}{c}0 \pm 0 \\
\mathrm{Ca}\end{array}$ & $\begin{array}{c}0 \pm 0 \\
\mathrm{Ba}\end{array}$ & $\begin{array}{c}0 \pm 0 \\
\mathrm{Ba}\end{array}$ & $\begin{array}{c}\mathrm{F}_{2,447}=- \\
\mathrm{P}=-\end{array}$ \\
\hline Control & $\begin{array}{c}1.4 \pm 0.12 \\
\mathrm{Ab}\end{array}$ & $\begin{array}{c}1.82 \pm 0.14 \\
\mathrm{Aa}\end{array}$ & $\begin{array}{c}0.78 \pm 0.08 \\
\text { Ac }\end{array}$ & $\begin{array}{c}\mathrm{F}_{2,447}=69.04 \\
\mathrm{P}<0.0001\end{array}$ \\
\hline Positive control & $\begin{array}{c}0.37 \pm 0.04 \\
\mathrm{Ba}\end{array}$ & $\begin{array}{c}0.06 \pm 0.02 \\
\mathrm{Bb}\end{array}$ & $\begin{array}{c}0.05 \pm 0.01 \\
\mathrm{Ab}\end{array}$ & $\begin{array}{c}\mathrm{F}_{2,447}=40.52 \\
\mathrm{P}<0.0001\end{array}$ \\
\hline $\mathrm{P}$ and $\mathrm{F}$ & $\begin{array}{c}\mathrm{F}_{4.745}=79.33 \\
\mathrm{P}<0.0001\end{array}$ & $\begin{array}{c}\mathrm{F}_{4.745}=158.89 \\
\mathrm{P}<0.0001\end{array}$ & $\begin{array}{c}\mathrm{F}_{4.745}=10.01 \\
\mathrm{P}<0.0001\end{array}$ & \\
\hline
\end{tabular}

Synthetic pesticides have caused many serious economical and environmental problems due to their broad spectrum toxicity. Therefore eucalyptus essential oil compounds were investigated as a natural alternative to be used against storage pests, pathogenic fungi and weeds at different concentrations and in 1-7 days, in vitro. The tested essential oil (E. camaldulensis Dehnh.) was very effective against all the insect pests used in this study. The indicated a potential for this essential oil to be used to control these storage pests.

Essential oils can easily be obtained from plant materials by vapor distillation method. They are preferable because they exhibit low toxicity for mammals, while are highly toxic to storage pests [27]. In earlier studies, toxic effects of some essential oils were assessed to determine possible fumigant, contact and ingestion activity against $R$. dominica, S. oryzae and T. castaneum [28]. It was reported that the essential oils obtained from Chenopodium ambrosioides leaves showed high insecticidal toxicity against Prostephanus truncatus, Callosobruchus chinensis, C. maculatus, A. obtectus and S. granarius [8]. In 
another study, the toxic effects of essential oils of Lavandula angustifolia, Rosmarinus officinalis, Thymus vulgaris and Laurus nobilis were observed to be effective against stored pests. The group has reported that 1,8-cineole, thymol and borneol were toxic at high doses on $S$. oryzae after 24 hours (at $0.1 \mu \mathrm{L} / 720 \mathrm{~mL}$ ) and $100 \%$ of the main components of camphor and linalool applied on $R$. dominica and $T$. castaneum caused approximately $20 \%$ of deaths [29]. The greatest fumigant toxicity against A. obtectus was seen with $F$. vulgare essential oil, followed by $T$. spicata and $L$. stoechas essential oils. The main components of plant essential oils of three plants showing high response were determined by GC-MS analysis. $F$. vulgare essential oil's main components were anisole $(79 \%)$ and L-fenchone $(13 \%)$. T. spicata and L. stoechas contain L-fenchone $(55 \%, 57 \%)$, camphor $(24 \%, 24 \%)$ and 1,8-cineole $(13 \%, 13 \%)$, respective. Main components L-fenchone and camphor caused about $100 \%$ mortality at $80 \mu \mathrm{L} / \mathrm{L}$ dose in 48 hours. The results indicate that $F$. vulgare essential oil or its components may have a potential for controlling of $A$. obtectus [30]. A. obtectus on the other hand was declared to be the most tolerant species against the essential oils [31]. As reported by these researchers were similar results with the present study.

In a previous investigation, the mycelial growth of most fungi used in the study, was affected by the essential oil which indicates the potential of this oil and its inhibitory effect against some important pathogenic fungi. It was found that four species of eucalyptus essential oil had inhibitory effect on some fungi; such as T. cucumeris $100 \%$ at $5 \mathrm{mg} / \mathrm{mL}, F$. oxysporum more than $84 \%$ at $5 \mathrm{mg} / \mathrm{mL}$ and C. globosum $100 \%$ at $10 \mathrm{mg} / \mathrm{mL}$ [11]. In an another report, $\beta$-citronellol, nerol, menthol, terpinen-4-ol, $\alpha$-terpineol, carvone, borneol compounds and commercial benomyl were determined as antifungal compounds, and a high concentration of $E$. camaldulensis was found to cause a remarkable inhibition against pathogenic fungi $F$. solani [15]. Of all the compounds in another study, Thymol was pointed the most strong antifungal compound against the four fungi (F. oxysporum, R. solani, A. niger and P. digitatum) [24]. When phenols, alkaloids and terpenes were extracted from E. camaldulensis and applied to the fungi, the results showed that terpene extract was the most active against fungi and alkaloids extract had less antifungal activity where the percentage of mycelial radial growths calculated as 99.55 and $72.44 \%$ respectively [25]. Additionally, in a study where Myrtus communus volatile oil was used against 19 phytopathogenic fungi, the effect of antifungal activity was determined as 10-100\% [26]. Essential oil of E. camaldulensis was shown to inhibit mycelial growth of fungi, $F$. oxysporum, $F$. verticillioides, $F$. solani, $F$. subglutinans and F. proliferatum. It was observed as effective at 7,8 and $10 \mu \mathrm{L} / \mathrm{mL}$ on the $5^{\text {th }}$ day [32]. The results revealed that E. camaldulensis leaf oils provided $100 \%$ inhibition of the mycelial growth of Thanatephorus cucumeris $(5 \mathrm{mg} / \mathrm{mL})$, and Chaetomium globosum $(10 \mathrm{mg} / \mathrm{mL})$. No inhibition effect was observed against $R$. oryzae even at the concentration of $10 \mathrm{mg} / \mathrm{mL}$ [33]. Eucalyptus of essential oil presented high antifungal activity against $S$. sclerotiorum and Colletotrichum circinans fungus species at 10 and $50 \mu \mathrm{L} / \mathrm{petri}$, but was not found effective against $F$. oxysporum, Alternaria mali and Botrytis cinerea in vitro conditions [34].

In this study, eucalyptus essential oil was ineffective on root and stem growth of $C$. arvensis at 5 , 10 and $20 \mu \mathrm{L}$ concentrations in 7 days, while it was effective on root growth of M. officinalis and A. retroflexus at the same concentrations in 7 days. In other studies, the following results were obtained, the herbicidal activity of E. globulus essential oil was also determined and the viabilities of A. blitoides, A. viridis and $C$. dactylon were found to be significantly lower than the control group [35] Eucalyptus tereticornis essential oil on the other hand, was reported to inhibit the germination of A. viridis [36]. The herbicidal effects of the oils on the seed germination and seedling growth of A. retroflexus, $C$. album, $L$. serriola and $R$. crispus were also determined [37]. The essential oil of Nepeta meyeri inhibited the germination of the seeds of weed species including A. retroflexus L., C. album L., C. arvense L. and $S$. arvensis L. [38].

Our results suggest that E. camaldulensis essential oil might have potential to be used as a natural insecticide, fungicide, as well as herbicide.

\section{ORCID}

Tamer Üstüner: 0000-0003-3584-4249

Şaban Kordali: 0000-0001-5669-5831

Ayşe Usanmaz Bozhüyük: 0000-0003-2450-6850

Memiş Kesdek: 0000-0001-9881-4050 


\section{References}

[1] J. Fletcher, C. Bender, B. Budawle, W.T. Cobb, S.E. Gold, C.A. Ishimaru, D. Luster, U. Melcher, R. Murch, H. Scherm, R.C. Seen, J.L. Sherwood, B.W. Sobral and S.A. Tolin (2006). Plant pathogen forensics: Capabilities needs, and recommendations, Microbiol. Mol. Biol. Rev. 70, 450-471.

[2] M. Rassaeifar, N. Hosseini, N. Haji Hasani Asl, P. Zandi and A. M. Aghdam (2013). Allelopathic effect of Eucalyptus globulus essential oil on seed germination and seedling establishment of Amaranthus blitoides and Cynodon dactylon, Trakia J. Sci. 1, 73-81.

[3] M. Verdeguer, M.A. Blazquez and H. Boira (2009). Phytotoxic effects of Lantana camara, Eucalyptus camaldulensis and Eriocephalus africanus essential oils in weeds of Mediterranean summer crops, Biochem. Syst. Ecol. 37, 362-369.

[4] H. Sodaeizadeh and Z. Hosseini (2012). Allelopathy an environmentally friendly method for weed control, International Conference on Applied Life Sciences, Turkey, 387-392.

[5] N. Dudai, A. Poljakoff-Mayber, A.M. Mayer, E. Putievsky and H.R. Lerner (1999). Essential oils as allelochemicals and their potential use as bioherbicides, J. Chem. Ecol. 25, 1079-1089.

[6] S.O. Duke, F.E. Dayan, J.G. Romagni and A.M. Rimando (2000). Natural products as sources of herbicides: current status and future trends, Weed Res. 40, 99-111.

[7] S. Kordali, R. Kotan and A. Cakir (2007). Screening of in-vitro antifungal activities of 21 oxygenated monoterpenes as plant disease control agents, Allelopathy J. 19, 373-392.

[8] A.L. Tapondjou, C. Adler, H. Bouda and D.A. Fontem (2002). Efficacy of powder and essential oil from Chenopodium ambrosioides leaves as post-harvest grain proctentants against six-stored product beetles, $J$. Stored Prod. Res. 38, 395-402.

[9] D.P. Papachristos and D.C. Stamopoulos (2002). Toxicity of vapours of three essential oils to the immature stages of Acanthoscelides obtectus (Say) (Coleoptera: Bruchidae), J. Stored Prod. Res. 38, 365-373.

[10] M. Bittner, M.E. Casanueva, C. Arbert, M.A. Aguilera, V.J. Hernández and J.V. Becerra (2008). Effects of essential oils from five plants species against the granary weevils Sitophilus zeamais and Acanthoscelides obtectus (Coleoptera), J. Chil. Chemi. Soc. 53, 1455-1459.

[11] Y.C. Su, C.L. Ho, E.I.C. Wang and S.T. Chang (2006). Antifungal activities and chemical compositions of essential oils from leaves of four eucalypts, Taiwan J. For. Sci. 21(1), 49-61.

[12] I. Somda, V. Leth and P. Sereme (2007). Antifungal effect of Cymbopogon citratus, Eucalyptus camaldulensis and Azadirachta indica oil extracts on Sorghum seed-borne fungi, Asian J. Plant Sci. 6(8), 1182-1189.

[13] N. Katooli, R. Maghsodlo and S.E. Razavi (2011). Evaluation of eucalyptus essential oil against some plant pathogenic fungi, J. Plant Breed. Crop Sci. 3(2), 41-43.

[14] U. Bashir and J.J. Tahira (2012). Evaluation of Eucalyptus camaldulensis against Fusarium solani, Int. J. Agric. Biol. 14, 675-677.

[15] A. Moradshahi, H. Ghadiri and F. Ebrahimikia (2003). Allelopathic effects of crude volatile oil and aqueous extracts of Eucalyptus camaldulensis Dehnh. leaves on crops and weeds, Allelopathy J. 12, 189-195.

[16] S. Kordali, A. Cakir and S. Sutay (2007b). Inhibitory effects of monoterpenes on seed germination and seedling growth, Z. Naturforsch. 62 (3-4), 207-214.

[17] A. Elaissi, H. Medini, M.L. Khouja, M. Simmonds and F. Lynen (2011). Variation in volatile leaf oils of five Eucalyptus species harvested from Jbel Abderrahman Arboreta (Tunisia), Chem. Biodivers. 8, 352-361.

[18] A.A. Shammam and A.M. Ghanuni (2011). Effect of ground leaves of Eucalyptus camaldulensis on the germination and growth of Cyperus rotundus L., Afri. J. Biol. Sci. 7, 47-52.

[19] R. Ataollahi, M. Dejam and S.S. Khalegh1 (2014). Phytotoxic effects of Eucalyptus globulus leaf extract on Solanum nigrum, South West. J. Hortic. Biol. Environ. 5 (1), 43-53.

[20] A.M. Ghanuni, A. Elshebani, M.A. Moftah and A.N. Lajili (2015). Allelopathic effect of (Eucalyptus camaldulensis) on peanut (Arachis hypogaea) crop and purple nutsedge (Cyperus rotundus) weed, Scholarly J. Agric. Sci. 5(6), 189-194.

[21] S. Kordali, A. Cakir, T.A. Akcin, E. Mete, A. Akcin, T. Aydın and H. Kilic (2009). Antifungal and herbicidal properties of esential oils and $n$-hexane extracts of Achillea gypsicola Hub-Mor. and Achillea biebersteinii Afan. (Ateraceae), Ind. Crop Prod. 29(2-3), 562-570.

[22] R.P. Adams (2007). Identification of essential oil components by Gas Chromatography/MassSpectrometry, Allured Publishing Corp, Carol Stream, Illinois.

[23] N. Dudai, D. Chaimovitsh, O. Larkov, R. Fischer, Y. Blaicher and A. Mayer (2009). Allelochemicals released by leaf residues of Micromeria fruticosa in soils, their uptake and metabolism by inhibited wheat seed, Plant Soil. 314, 311-317.

[24] G.I.K. Marei, M.A. Abdel Rasoul and S.A.M. Abdelgaleil, (2012). Comparative antifungal activities and biochemical effects of monoterpenes on plant ptahogenic fungi, Pestic. Biochem. Physiol. 103, 56-61.

[25] A.J. Fradi and A.M.Y. Al-Araji (2015). Effect of Eucalyptus camaldulensis terpenes, alkaloids and phenols against Fusarium oxysporum, Iraqi J. Sci. 56 (4), 2807-2810. 
[26] S. Kordali, A. Usanmaz, A Cakir, A. Komaki and S. Ercisli (2016). Antifungal and herbicidal effects of fruit essential oils of four Myrtus communis Genotypes, Chemis. Biodiver. 13(1), 77-84.

[27] E. Shaaya, U. Ravid, N. Paster, B. Juven, U. Zisman and V. Pissarev (1991). Fumigant toxicity of essential oils against four major stored product insects, J. Chem. Ecol. 17, 499-504.

[28] B.H. Lee, P.C. Annis, F. Tumaalii and S.E. Lee (2004). Fumigant toxicity of Eucalyptus blakelyi and Melaleuca fulgens essential oils and 1,8- cineole against different developmental stages of the rice weevil Sitophilus oryzae, Phytoparasitica, 32, 498-506.

[29] V. Rozman, I. Kalinovic and Z. Korunic (2007). Toxicity of naturally occuring compounds of Lamiaceae and Lauraceae to three stored-product insects, J. Stored Prod. Res. 43, 349-355.

[30] T. Selimoglu, A. Gökce and D. Yanar (2015). Fumigant toxicities of some plant essential oils on A. obtectus Say (Coleoptera: Bruchidae), J. Turk Ento. 39 (1), 109-118.

[31] A. Ayvaz, O. Sagdic, S. Karaborklu and I. Ozturk (2010). Insecticidal activity of the essential oils from different plants against three stored-product insects, J. Insect Sci. 10(21), 1-13.

[32] M.M. Gakuubi, A.W. Maina and J.M. Wagacha (2017). Antifungal activity of essential oil of Eucalyptus camaldulensis Dehnh. against selected Fusarium spp., Inter. J. Microbiol. 2017, 1-7.

[33] P. Siramon, Y. Ohtani and H. Ichiura (2013). Chemical composition and antifungal property of Eucalyptus camaldulensis leaf oils from Thailand, Rec. Nat. Prod. 7(1), 49-53.

[34] R. Kocak and N. Boyraz (2006). Fungicidal and fungistatic effects of essential oils of some plants. Selcuk University, Agri. Faculty J. 20 (38), 76-81.

[35] D.R. Batish, N. Setia, H. Singh and R.K. Kohli (2004). Phytotoxicity of lemon-scented eucalypt oil and its potential use as a bioherbicide, Crop Pro. 23(12), 1209-1214.

[36] S. Kaur, H.P. Singh, D.R. Batish and R.K. Kohli (2011). Chemical characterization and allelopathic potential of volatile oil of Eucalyptus tereticornis against Amaranthus viridis, J. Plant Inter. 6(4), 297-302.

[37] N. Nishida, S. Tamotsu, N. Nagata, C. Saito and A. Sakai (2005). Allelopathic effects of volatile monoterpenoids produced by Salvia leucophylla: Inhibition of cell proliferation and DNA synthesis in the root apical meristem of Brassica campestris seedlings, J. Chem. Ecol. 31(5), 1187-1203.

[38] S. Kordali, A. Tazegul and A. Cakir (2015). Phytotoxic effects of Nepeta meyeri Benth. extracts and essential oil on seed germinations and seedling growths of four weed species, Rec. Nat. Prod. 9(3), 404-418.

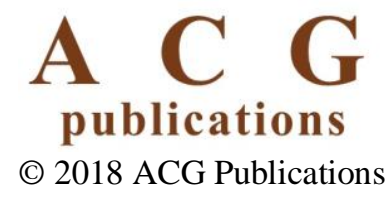

